\title{
The numerical simulation of compressible jet at low Reynolds number using OpenFOAM
}

\author{
Andrey Epikhin ${ }^{1,3, *}$, Matvey Kraposhin ${ }^{1,2,4}$, and Kirill Vatutin ${ }^{1,4}$ \\ ${ }^{1}$ Ivannikov Institute for System Programming of the RAS, 109004, Solzhenitsyna str., 25, Moscow, Russia \\ ${ }^{2}$ Keldysh Institute of Applied Mathematics of the RAS, 125047, Miusskaya pl., 4, Moscow, Russia \\ ${ }^{3}$ Bauman Moscow State Technical University, 105005, 2-aya Baumanskaya str., 5, Moscow, Russia \\ ${ }^{4}$ Department of Aeromechanics and Flight Engineering of MIPT, 140180, Gagarina str., 16, Zhukovsky, Russia
}

\begin{abstract}
The paper presents an analysis of various approaches for calculation gas-dynamic parameters and acoustic perturbations generated by a compressible jet at low Reynolds number $(M=0.9, R e=3600)$. The jet flow parameters at selected conditions are well studied and can be used for validation of the numerical methods and schemes. The OpenFOAM software package with various approaches (solvers) such as pimpleCentralFoam, dbnsTurbFoam and new QGDFoam solver based on QGD-algorithms are considered. The results of time-averaged flow parameters and acoustic properties are compared with the experimental data. To determine the acoustic perturbation the Ffowcs Williams and Hawkings analogy implemented in our OpenFOAM library (libAcoustic) has been used.
\end{abstract}

\section{Introduction}

Aero-acoustics problems are important and relevant to different engineering applications. In particular, one of such problems is the reduction of acoustic noise arising due to the interaction of turbulent flows with a fluid, for example, when a rocket engine jet is ejected into the atmosphere. The spatial structure arising from such underexpanded jet flows is characterized by the development of the Kelvin-Helmholtz instability waves, which lead to drain of energy in the radial direction. Their further development is accompanied by the formation of large-scale and small-scale vortex structures, which lead to the occurrence of acoustic noise. Therefore, numerical simulation of the jet flows process at large Reynolds numbers and the determination of acoustic disturbances generated by them presents considerable computational difficulties. In turn, the flow parameters of the jets with low Reynolds numbers are well studied [1-3] and can be used to validate the numerical method and schemes, since there is no need to use a very detailed grid and additional assumptions, related with different turbulence models.

Nowadays, most of the industrial problems of acoustic noise research are solved using commercial software packages. An alternative to them is the development of open source packages, for example, OpenFOAM, which allows to solve a wide range of continuum mechanics problems not only using standard solvers and utilities but also to improve them. The most common approaches for solving jet flows include methods based on solving the Riemann problem using the following approximations that are part of the dbnsTurbFOAM solver: Local LaxFriedrichs (Rusanov), Roe, Harten-Lax-Leer (HLL), HLL-Contact (HLLC). However, these schemes poorly describe flows at Mach numbers $M<0.5$. Such methods as SIMPLE, PISO, PIMPLE are verified for weakly compressible flows, but they are ineffective in trans and supersonic regimes. As a result, the pimpleCentralFoam solver based on a hybrid approximation method for convective terms and the Kurganov-Tadmor scheme was proposed [4]. Another alternative to these approaches is to use the method based on quasi-gas dynamics (QGD) algorithms $[5,6]$. This approach uses the approximation of regularized or quasi-gas dynamics equations and is implemented in the QGDFoam solver [7,8].

Below are the results of the numerical simulation of the jet flow from a circular nozzle with $M=0.9$ and $R e=3600$, obtained using the above approaches. To determine acoustic disturbances, we use the Ffowcs Williams and Hawkings analogy, which is implemented in the libAcoustic library based on the OpenFOAM package $[9,10]$. The flow parameters and geometry match an experimental study conducted by Stromberg et al. [1] and results are compared to their data whenever possible.

\section{Numerical setup}

The subject of the present study is round jet with exit Mach number $M=0.9$, constant stagnation temperature $T_{0}=297 \mathrm{~K}$ and a Reynolds number, based on jet exit conditions $R e=\rho_{j} U_{j} D / \mu_{j}=3600$. The following initial condition were considered for the problem of spatial jet flow: nozzle exit diameter $D=0.0079 \mathrm{~m}$, jet exit velocity $U_{j}=286 \mathrm{~m} / \mathrm{s}$, jet exit temperature $T_{j}=256 \mathrm{~K}$, jet exit pressure $p_{j}=1823 \mathrm{~Pa}$, chamber pressure $p_{c}=p_{i}$, ambient pressure $p_{a}=101325 \mathrm{~Pa}$.

* Corresponding author: andrey.epikhin@bk.ru 
The computational domain presents a rectangular parallelepiped in which the output boundary is removed by $60 D$, the side edges - by $21 D$ (Fig. 1).

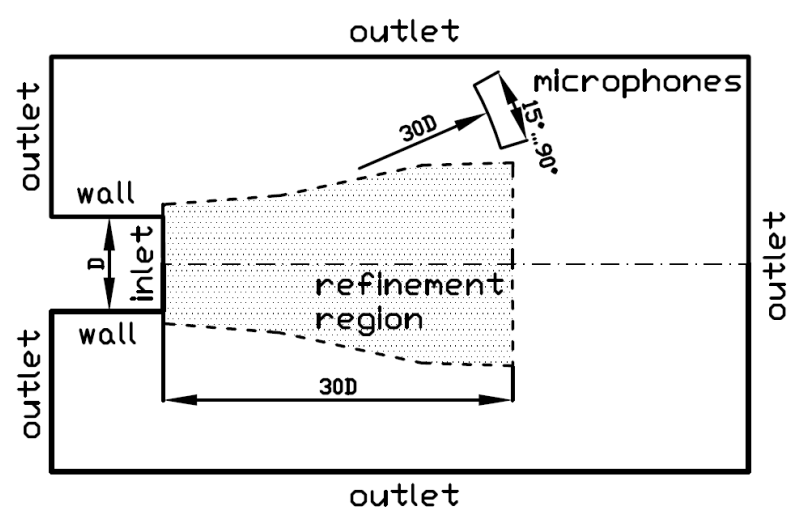

Fig. 1. Scheme of the boundary conditions.

The inflow boundary correspond to the cut of the circular nozzle and coincides with the origin. The following mean streamwise velocity profile is imposed on the inflow boundary:

$$
U(r)=0.5 \cdot U_{j} \cdot\left[1+\tanh \left(10 \cdot\left(1-\frac{2 r}{D}\right)\right)\right] .
$$

This condition is determine a thin laminar annular shear layer in the exit of nozzle with a mean thickness parameter equal to 10. SnappyHexMesh is used to make various of computational grids with different numbers of cells per nozzle exit diameter $(\mathrm{CPD})$ : 2.8 million $(\mathrm{CPD}=10)$; 13 million $(\mathrm{CPD}=30) ; 20$ million $(\mathrm{CPD}=40) ; 33$ million cells $(\mathrm{CPD}=50)$. Additionally, the computational grid is refined $30 D$ downstream. Figure 2 shows a fragment of the computational grid.

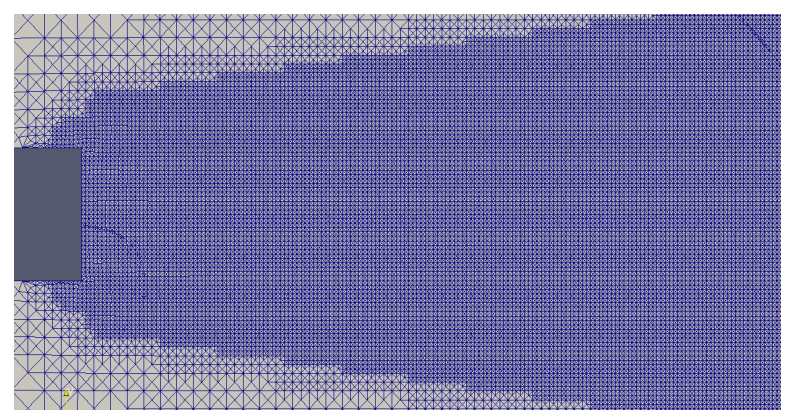

Fig. 2. The fragment of the computational grid.

The turbulence model is not applied. Various computational grids and the following solvers are used for numerical simulation:

- dbnsTurbFoam (DBNS) with the HLLC scheme;

- pimpleCentralFoam (PCF) with vanLeer and Minmod schemes;

- QGDFoam (QGD) with $\alpha_{Q G D}=0.05 \ldots 0.3$.

\section{Results and discussion}

Figure 3 shows the jet vortex structure obtained by pimpleCentralFoam solver with grid resolution $40 \mathrm{CPD}$.

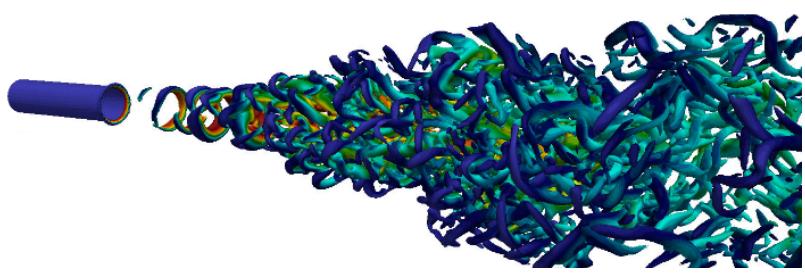

Fig. 3. Instantaneous iso-surface of $Q$-criteria.

Figure 4 shows the axial distribution of the jet mean Mach number in comparison with the experimental data [1] for different approaches and computational grids.

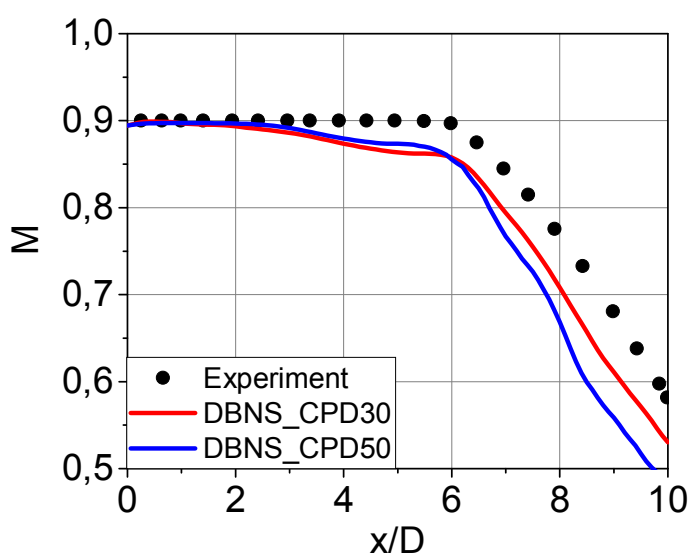

a)

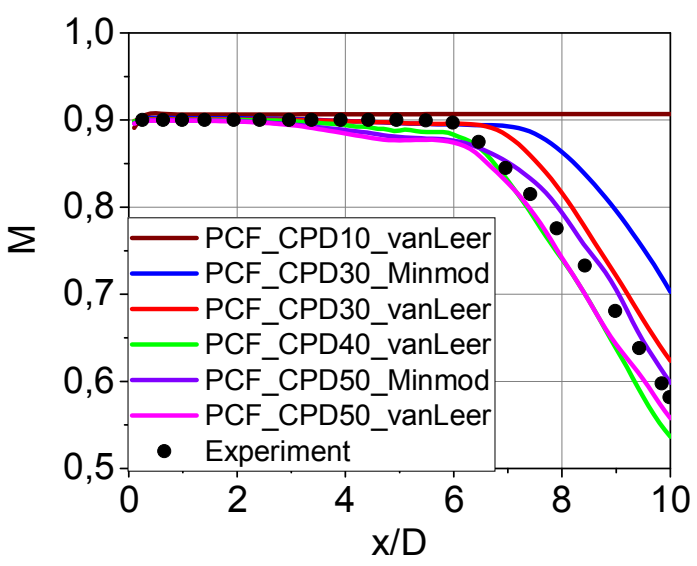

b)

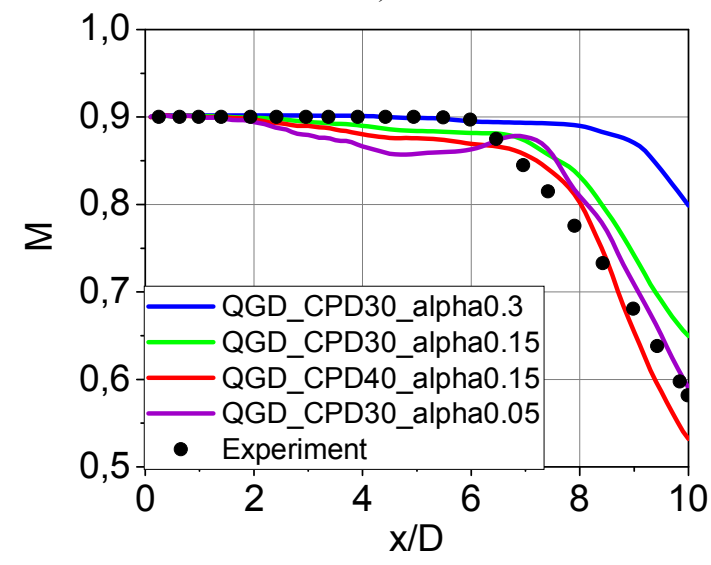

c)

Fig. 4. Axial distribution of centerline mean Mach number for various approaches: a) DBNS; b) PCF; c) QGD. 
The complex research of the pulsating components of the flow is performed by calculating acoustic noise in the far field using the Ffowcs Williams and Hawkings analogy (Farassat's Formulation 1A [11]). Virtual microphones are located at the arc of radius 30 jet diameters centred at the nozzle exit for the angles from the jet axis equal from 15 to 90 degrees. Based on the recommendations from $[12,13]$ on the choice of the shape of the control surface in the calculation of jet streams, two open surfaces are put around jet as illustrated in Figure 5 (s1 - red line, s2 - blue line). The control surfaces start $0.1 D$ downstream of the inflow boundary and extend to $35 D$ along the streamwise direction.

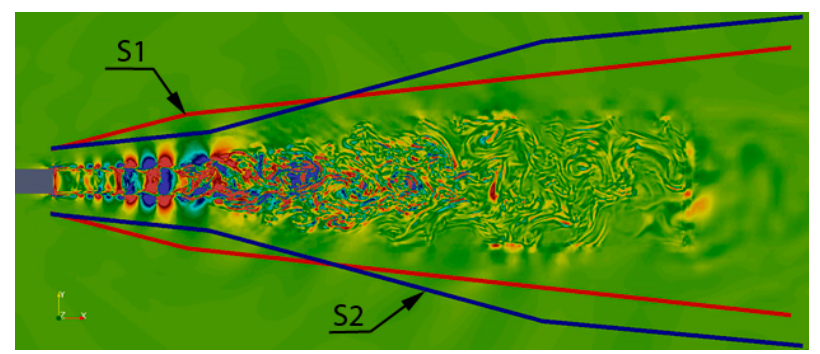

Fig. 5. Schematic showing the 2 open control surfaces surrounding the jet flow. (Divergence of velocity contours are shown).

The overall sound pressure level (OASPL) for this case is calculated in terms of a reference pressure scaled to the ambient pressure:

$$
\begin{aligned}
& p_{r e f}=2 \cdot 10^{-5} \cdot p_{c} / p_{a}, \\
& O A S P L=20 \cdot \log _{10}\left(p_{r m s} / p_{r e f}\right) .
\end{aligned}
$$

The OASPL directivity distribution for PCF solver is presented in Figure 6.

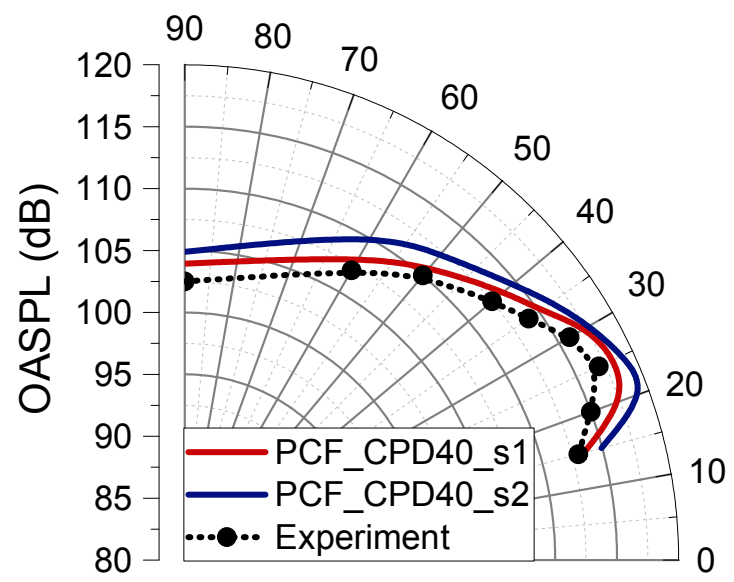

Fig. 6. Sound pressure level directivity distributions for $M=0.9$, $R e=3600$ and PCF solver with different control surfaces.

The results show that the minimum grid resolution required for the numerical calculation of the process of formation and development of instabilities in space is more than 30 CPD. The Minmod scheme is more stable than the vanLeer scheme but less accurate. Numerical simulations with different values of $\alpha_{Q G D}$ shows possibility of modelling QGD numerical solution by tuning parameter without consuming additional computational resources due to mesh refinement. It can be noted that the behaviour of the QGD algorithm with $\alpha_{Q G D}=0.3$ is similar to the use of the Minmod scheme for the hybrid approach, and for $\alpha_{Q G D}=0.15$ is similar to vanLeer scheme.

\section{Conclusions}

The study of different numerical simulation approaches of jet flow is carried out and gas-dynamic and acoustic parameters obtained at $M=0.9, R e=3600$ are compared with experimental data. Results allowed to formulate recommendations for the calculation of jet streams at low Reynolds numbers $(R e<4000)$. The resolution of the grid should be no less than 30-40 CPD. The approach based on solving the Riemann problem using approximations does not allow to calculate such process correctly. In turn, the hybrid approach with the vanLeer scheme and the method based on QGD algorithms, with $\alpha_{Q G D}=0.15$ makes it possible to simulate the process of formation and development of instabilities in space and to determine acoustic disturbances.

\section{Acknowledgments}

This work was supported by the RSF under the grant No. 19-11-00169. The results of the work were obtained using computational resources of MCC NRC «Kurchatov Institute», http://computing.nrcki.ru. The research is carried out using the equipment of the shared research facilities of HPC computing resources at Lomonosov Moscow State University.

\section{References}

1. J.L. Stromberg, D.K. McLaughlin, T.R. Troutt, J. Sound Vib., 72(2), 159-176, (1980)

2. G.L. Morrison, D.K. McLaughlin, AIAA J., 18(7), 793800, (1980)

3. T.R. Troutt, D.K. McLaughlin, J. Fluid Mech., 116, 123156, (1982)

4. M.V. Kraposhin, M. Banholzer, M. Pfitzner, I.K. Marchevsky, Int. J. Numer. Meth. Fl., 88(2), 79-99, (2018)

5. T.G. Elizarova, Quasi-gas dynamic equations (Springer, Berlin, 2009)

6. B.N. Chetverushkin, Russ. J. Numer. Anal. M., 20(4), 337-351, (2005)

7. https://github.com/unicfdlab/QGDsolver

8. M.V. Kraposhin, D.A. Ryazanov, E.V. Smirnova, T.G. Elizarova, M.A. Istomina, Proceedings-2017 Ivannikov ISPRAS Open Conference, 117-123, (2018)

9. https://github.com/unicfdlab/libAcoustics

10. A. Epikhin, I. Evdokimov, M. Kraposhin, M. Kalugin, S. Strijhak, Procedia Comput. Sci., 66, 150-157, (2015)

11. K.S. Brentner, F. Farassat, AIAA J., 36(8), 1379-1386, (1998)

12. A. Uzun, A.S. Lyrintzis, G.A. Blaisdell, AIAA Paper, 4982-5001, (2004)

13. M. Shur, P. Spalart, M. Strelets, Int. J. Aeroacoust., 4, 213-246, (2005) 